\title{
Ordering silver nanowires for chiroptical activity
}

\author{
Xueru Guo ${ }^{1 \dagger}$, Dan $\mathrm{Wu}^{2 \dagger}$, Yi Li ${ }^{1 \dagger}$, Zhen $\mathrm{He}^{1}$, Jin-Long Wang ${ }^{1}$, Chong Zhang ${ }^{1}$, Zhao Pan ${ }^{1}$, Yuanjie Pang ${ }^{2 *}$, \\ Taotao Zhuang ${ }^{1^{*}}$ and Shu-Hong $\mathrm{Yu}^{1^{*}}$
}

\begin{abstract}
With the development of chiral materials, remarkable progress has been made in the application fields of biology, chemistry, and physics in the past decades. Here we develop a solo-Ag-nanowire (NW)-based, bottom-up chiral construction strategy to fabricate a chiral plasmonic film with strong chiroptical activity in the purple-blue band. Finitedifference time-domain simulations predict that helical structures made by Ag NWs with certain interlayer angles possess strong chiroptical intensity. We then apply a Langmuir-Blodgett technique to construct the layered NW assemblies with both handedness via regulating interlayer angle direction. The Ag NW assemblies show the interlayer-angleand film-thickness-dependent circular dichroism, reaching the highest anisotropic factor of 0.03 . These new chiral structures will serve as ideal candidates for chiral sensors thanks to their specific plasmonic properties.
\end{abstract}

Keywords: chiral assembly, finite-difference time-domain simulations, Ag nanowires, chiroptical activity

\section{INTRODUCTION}

Chirality, a descriptor of geometric property wherein the object cannot coincide with its mirror image, is a ubiquitous and intriguing phenomenon in the natural world. Chiral nanomaterials have been studied in the past years [1-5] and demonstrated applications in various fields including enantioselective separation, enantioselective catalysis, chiral sensing, and optoelectronic devices [6-10]. Among these fields, chiral sensing techniques have been designed to analyze the stereostructure of chiral molecules, but their signal strength still has a major gap as compared with practical demand [11,12]. It is highly desired, therefore, to develop related techniques for enhancing the signal intensity.

We here first focus on Raman optical activity (ROA) spectroscopic technique, one of the important chiral sensing technologies, depending on the difference of Raman scattering intensity between both handed circularly polarized light (CPL) [13]. We propose a chiral plasmonic structure with circular dichroism (CD) response and the effect of surface-enhanced ROA (SEROA), leading to increased sensitivity of ROA and decreased detection limit in the chiral sensing application [14].
In addition, a surface-enhanced Raman scattering-chiral anisotropy effect enables enhancing the detection sensitivity, which is based on selective resonance coupling between the chiral plasmonic modes of materials and the induced electric/magnetic dipoles of analytes [15]. This further highlights the great potentials of chiral plasmonic materials in chiral sensing.

Plasmonic nanostructures are competent for strong lightmatter interaction owing to their tunable local surface plasmonic resonance effect [16]. Ag has stronger plasmonic response in the shorter-wavelength regime (i.e., near-ultra-violet-visible), and benefits from a higher earth-availability and a lower cost compared with other noble metals [17]. One-dimensional (1D) Ag nanowires are especially attractive for strong optical response on account of their shape and size [17].

Recently, one bottom-up study used the way of grazing incidence spraying and layer-by-layer assembly to obtain chiral Ag via introducing polyelectrolyte [18]. However, building pure chiral Ag to realize high electroconductibility for final practical applications and uncovering the corresponding chirality generation process is still challenging. Here we utilized finite-difference time-domain (FDTD) simulations, providing a comprehensive understanding of the chiral formation mechanism, to predict solo chiral Ag construction without other additives. With a modified Langmuir-Blodgett (LB) method, we build a layer-aligned Ag NW assembly that brings large birefringence and linear dichroism, and thus obtain a chiral film with giant optical activity. Our initial trials further confirmed that the one-layer ordered Ag NW film, constructed via LB assembly technique, has a high intensity of linear dichroism (Fig. S1). By adjusting the interlayer angle and the thickness of films, we achieved a $g$ factor of up to 0.03 with an interlayer angle of $45^{\circ}$ using a two-layer assembled film. This work provides a potential way to utilize achiral Ag NWs to construct chiral plasmonic films.

\section{EXPERIMENTAL SECTION}

\section{FDTD simulation}

Electromagnetic simulations of $\mathrm{CD}, g$ factor spectra, and electric field distribution were carried out using a commercial FDTD software package (Ansys Lumerical FDTD). After a convergence test on mesh size using 2-, 1- and 0.5-nm meshes, respectively,

\footnotetext{
${ }^{1}$ Division of Nanomaterials \& Chemistry, Hefei National Laboratory for Physical Sciences at the Microscale, Institute of Energy, Hefei Comprehensive National Science Center, CAS Center for Excellence in Nanoscience, Department of Chemistry, Institute of Biomimetic Materials \& Chemistry, Anhui Engineering Laboratory of Biomimetic Materials, University of Science and Technology of China, Hefei 230026, China

${ }^{2}$ School of Optical and Electronic Information, Wuhan National Laboratory for Optoelectronics, Huazhong University of Science and Technology, Wuhan 430076, China

† These authors contributed equally to this work.

* Corresponding authors (emails: tzhuang@ustc.edu.cn (Zhuang T); yuanjie_pang@hust.edu.cn (Pang Y); shyu@ustc.edu.cn (Yu SH))
} 
the 1-nm mesh was used for simulation. Periodic boundary conditions were used in the Ag layer plane to represent an indefinitely extended Ag wire grid. The CD spectra were calculated by the transmission difference between left-handed circular polarized light (LCPL) and right-handed circular polarized light (RCPL). The $g$ factor spectra were calculated as the quotient of $\mathrm{CD}$ divided by absorption. The wavelength scan range was chosen from 200 to $600 \mathrm{~nm}$. The refractive index was obtained from the database of CRC Handbook of Chemistry and Physics; the polynomial fitting method in the software was adopted to fit the dielectric constant of the material (fit tolerance 0.1, max coefficients 8).

\section{Computing methods}

The relevant parameters were based on the formulas in the literature [19]. The dipole-dipole interaction was related to the energy between dipoles from two adjacent layers:

$V_{12}=\frac{p_{1} p_{2}}{n R_{12}^{3}}\left(\mathbf{e}_{1} \cdot \mathbf{e}_{2}-3\left(\mathbf{e}_{1} \cdot \mathbf{e}_{12}\right)\left(\mathbf{e}_{2} \cdot \mathbf{e}_{12}\right)\right)$,

where $p_{1}$ and $p_{2}$ mean two dipoles perpendicular to the direction of the silver nanowire, $\mathbf{e}_{1,2}=\mathbf{p}_{1,2} /\left\|\mathbf{p}_{1,2}\right\|, \mathbf{e}_{12}=\mathbf{r}_{12} /\left\|\mathbf{r}_{12}\right\|$, in which $\mathbf{r}_{12}$ is the vector joining the two dipoles. The resulting CD spectrum can be obtained by a parameter described by the angle-related rotational strength:

$R^{ \pm}= \pm \frac{\pi}{2 \lambda_{0}} \mathbf{r}_{12} \cdot\left(\mathbf{P}_{1} \times \mathbf{P}_{2}\right)$,

where $\lambda_{0}$ is the wavelength of the uncoupled local surface plasmon resonance.

\section{Chemicals}

$\mathrm{AgNO}_{3}$, polyvinylpyrrolidone (PVP, $M_{\mathrm{w}} \approx 40,000 \mathrm{~g} \mathrm{~mol}^{-1}$ ), glycerol, $\mathrm{NaCl}, \mathrm{NaBr}$, ethanol, chloroform, $\mathrm{N}, \mathrm{N}$-dimethylformamide (DMF) and ethylene glycol (EG) were all purchased from Sinopharm Chemical Reagent Co., Ltd. (China). All chemicals are of analytical grade and they were used as received without further purification.

\section{Synthesis of Ag NWs}

Ag nanowires with an average diameter of $60 \mathrm{~nm}$ were synthesized based on the previous work with minor modifications [20,21]. Firstly, $190 \mathrm{~mL}$ glycerol and $5.86 \mathrm{~g}$ PVP were added into a $500-\mathrm{mL}$ three-neck round flask, and heated to $100^{\circ} \mathrm{C}$ under stirring to obtain the homogeneous solution. After the temperature dropped to $30^{\circ} \mathrm{C}, 1.58 \mathrm{~g}_{\mathrm{AgNO}_{3}}$ was added to the solution. Then, the solution was heated from room temperature to $210^{\circ} \mathrm{C}$ under gentle stirring. During the heating process, when the temperature reached around $60^{\circ} \mathrm{C}$, a solution containing $59 \mathrm{mg} \mathrm{NaCl}, 0.5 \mathrm{~mL} \mathrm{H}_{2} \mathrm{O}$ and $10 \mathrm{~mL}$ glycerol was added to the solution. After reaching $210^{\circ} \mathrm{C}$, the heating was stopped immediately and then $200 \mathrm{~mL}$ deionized water was slowly added with a pipette gun to the solution. The final solution was kept undisturbed for $24 \mathrm{~h}$ to remove Ag nanoparticles. Finally, the upper liquid was poured out and the $\mathrm{Ag} \mathrm{NWs}$ at the bottom were dissolved in ethanol. Ag NWs with other diameters were synthesized according to the previous literature with minor modifications [22]. At room temperature, $\mathrm{NaBr}(0.1 \mathrm{~mL}$, $\left.110 \mathrm{mmol} \mathrm{L}^{-1}\right), \mathrm{NaCl}\left(0.2 \mathrm{~mL}, 210 \mathrm{mmol} \mathrm{L}^{-1}\right)$, PVP $(1.0 \mathrm{~mL}$, $\left.505.0 \mathrm{mmol} \mathrm{L}^{-1}\right)$ and fresh $\mathrm{AgNO}_{3}\left(1.0 \mathrm{~mL}, 265.0 \mathrm{mmol} \mathrm{L}^{-1}\right)$ in EG $(7.7 \mathrm{~mL})$ were added into a $50-\mathrm{mL}$ flask and placed in an oil bath. The solution was stirred for $30 \mathrm{~min}$ and heated to $170^{\circ} \mathrm{C}$ in
15 min under $\mathrm{N}_{2}$ protection, followed by 1-h reaction without stirring. The obtained Ag NW solution was purified by the slow addition of acetone. The supernatant was removed and the NWs with a mean diameter of $40 \mathrm{~nm}$ were aggregated at the bottom. We controlled the diameter of NWs by varying the amount of $\mathrm{NaBr}$ solution $\left(0.1 \mathrm{~mL}, 80 \mathrm{mmol} \mathrm{L}^{-1}\right.$ for $50 \mathrm{~nm}$, and 0 for $80 \mathrm{~nm}$ ) added in the reaction.

\section{Preparation of $\mathrm{Ag}$ NW film}

The Ag NW solution was washed twice with ethanol and then centrifuged. A certain amount of Ag NWs were taken and dissolved in DMF, followed by adding an equal volume of chloroform into the solution and shaking evenly. The formed solution was dropped onto the water surface, flatted in the LB machine, by a $100-\mathrm{mL}$ micro-syringe and assembled to a monolayer NW film. The film was transferred onto a quartz substrate and waited to dry. Then the substrate with one-layered film was rotated by a particular angle in a particular direction before transferring the next layer $[23,24]$.

\section{Characterization}

Transmission electron microscopy (TEM) was performed on an H7650 microscope (Hitachi, Japan) at $120 \mathrm{kV}$. Scanning electron microscopy (SEM) was performed on a Zeiss Supra 40 SEM operating at $5 \mathrm{kV}$. Scanning probe microscopy (AFM) was performed on a Di innova. Ultraviolet-visible spectroscopy (UVvis) absorption spectra were collected on a UV-2600 (Shimadzu Corporation, Japan) at room temperature. The polarizing transmission spectrometer was built with some commercial components by our lab. Optical performance measurement was performed on a CD spectrometer (JASCO-1500). For eliminating the possible angle-dependent interference caused by linear dichroism, the sample was rotated by several certain angles in the plane direction perpendicular to the optical path for tests. Image- $J$ software was used to represent the spatial distribution of NWs and the SEM images were colored according to the different spatial angles of NWs.

\section{RESULTS AND DISCUSSION}

We started with comprehensive FDTD simulations (ansys.com) to study how the Ag NW assembly realizes chirality. After a series of convergence tests (Fig. S2), we simulated the transmission intensity under incident light with different polarization angles to confirm the orderliness of ordered NWs (Fig. 1a). Then we simulated exemplary two-layered NW structures with interlayer angles of $15^{\circ}, 30^{\circ}, 45^{\circ}, 60^{\circ}, 75^{\circ}$, and $90^{\circ}$, respectively (Fig. 1b), to represent different Ag helical structures. For the two-layered, left-handed structure, the peaks of CD absorption spectra (Fig. 1c) and the asymmetry factor (Fig. 1d) were around at a wavelength of $350 \mathrm{~nm}$ for all interlayer angles, and the highest values occurred with an interlayer angle of $45^{\circ}$.

The simulated electric field intensities demonstrated negligible difference at all positions monitored (Fig. 2a-e and Figs S3-S5), we therefore turned to a comprehensive Born-Kuhn model towards chirality understanding [19,25]. Nanorods, nanoellipsoids, or in our case, gap plasmon between metal NWs, can be regarded as electric dipoles [19,26-28]. As the CPL passes through the first Ag NW layer and strikes the second one, the phase-incremented field and rotated NWs form two sets of dipoles. The angles between dipoles from neighboring layers dictate the energy level of the synergetic state, and the structure 

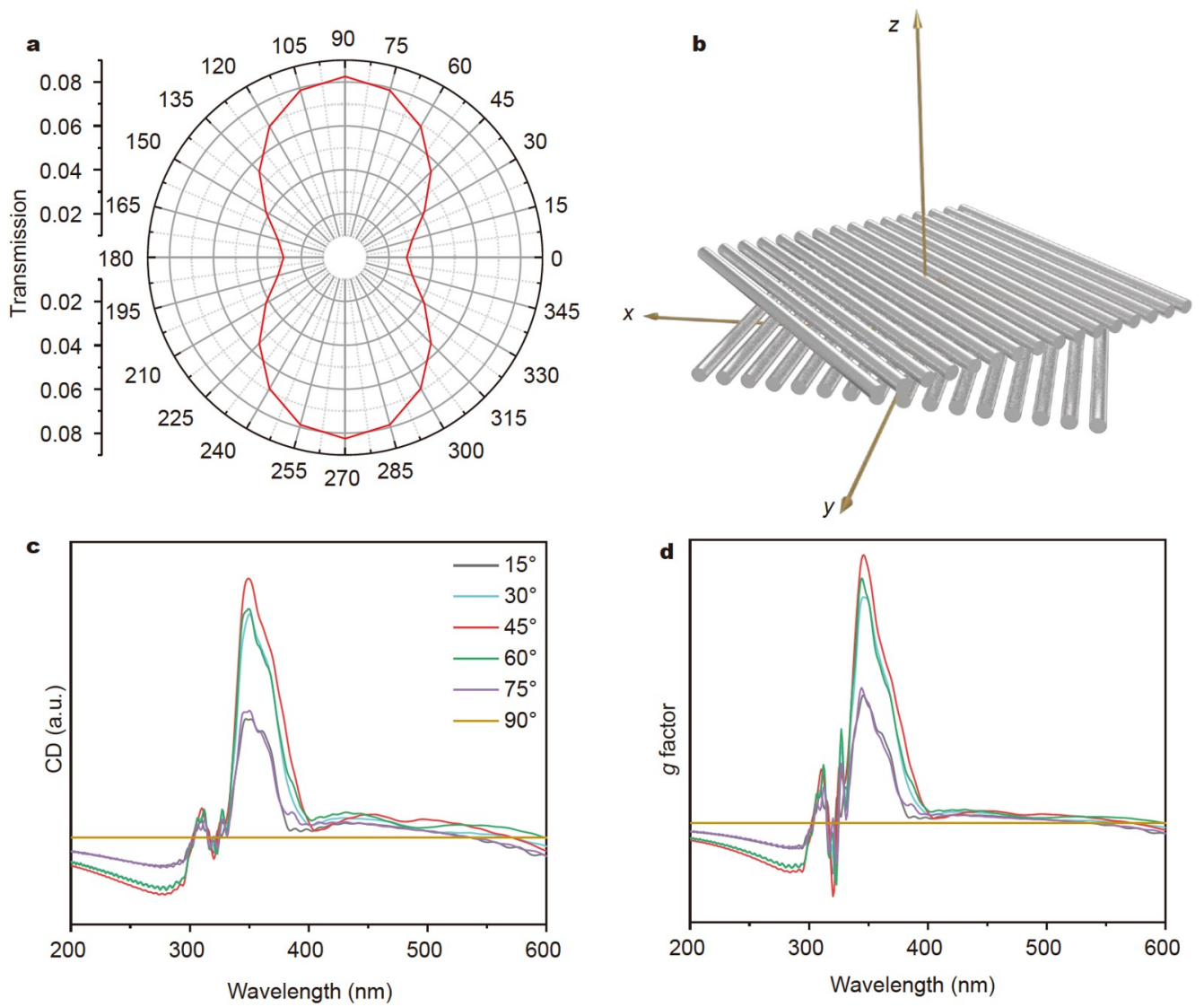

Figure 1 FDTD simulations of two-layer-aligned Ag NW films with different interlayer angles. (a) Polar diagram of simulated polarization-dependent transmission intensity of ordered one-layered films. (b) FDTD model of two-layered, left-handed films with tunable interlayer angles. (c, d) Calculated CD spectra (c) and corresponding $g$-factor results (d) of left-handed films with the angle of $15^{\circ}, 30^{\circ}, 45^{\circ}, 60^{\circ}, 75^{\circ}$ and $90^{\circ}$, respectively.
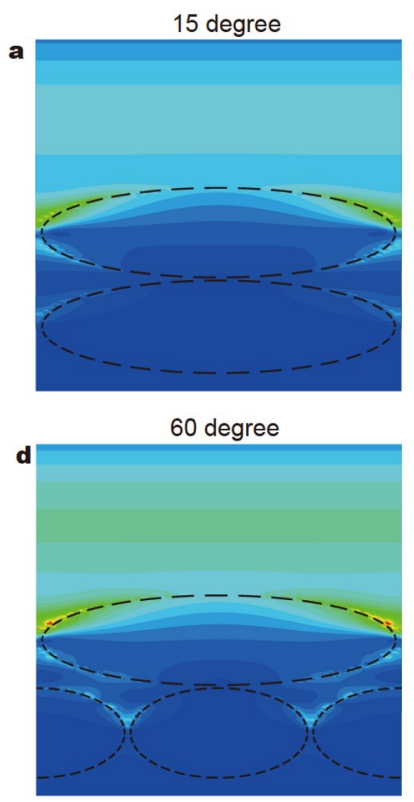
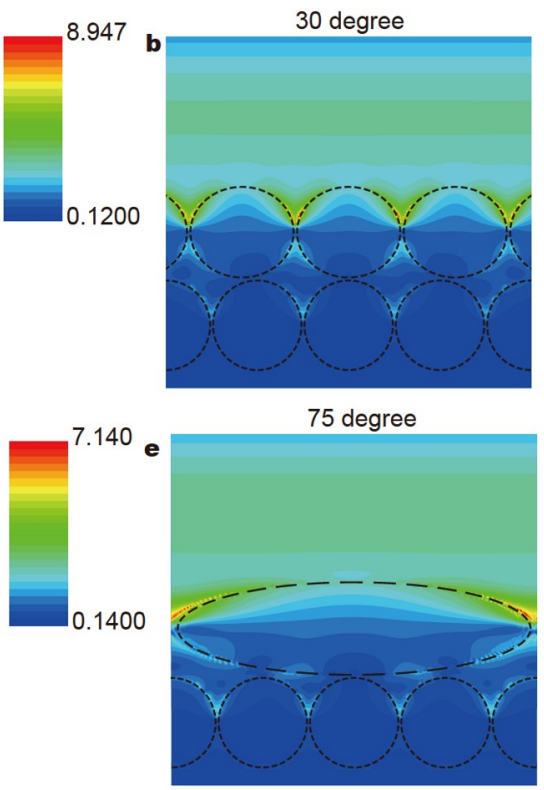
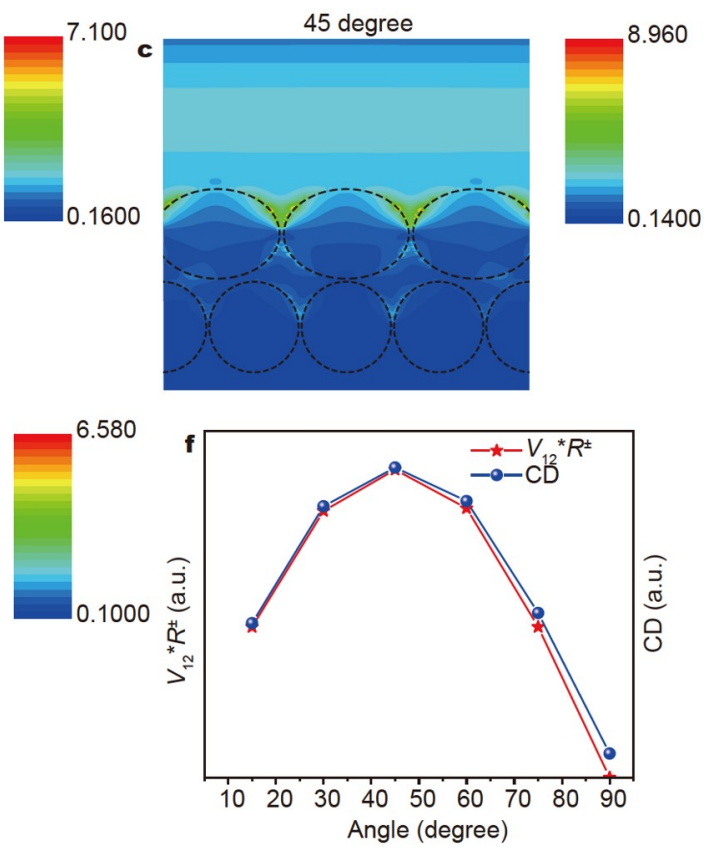

Figure 2 Simulated electric field intensity of two-layer-aligned Ag NW films with different interlayer angles. (a-e) Simulated electric field profiles at $Y=0$ plane for left-handed, two-layered films with interlayer angles of $15^{\circ}(\mathrm{a}), 30^{\circ}(\mathrm{b}), 45^{\circ}(\mathrm{c}), 60^{\circ}(\mathrm{d})$, and $75^{\circ}$ (e), respectively, excited by RCPL at $350 \mathrm{~nm},(\mathrm{dashed}$ line: outline of NWs). (f) Calculated $V_{12}{ }^{ \pm} R^{ \pm}$(red line) and CD intensity (blue line) with different interlayer angles. 
obtaining the largest energy difference under the excitation of LCPL and RCPL possesses the largest CD signal. For semiquantitative analysis (Table $\mathrm{S} 1$ ), the intensity of $\mathrm{CD}$ is proportional to $V_{12}{ }^{*} R^{ \pm}$, with $V_{12}$ being the energy difference between dipoles from two adjacent layers and $R^{ \pm}$being the interlayerangle-related rotational strength (Fig. 2f). As a result, films stacked with the interlayer angle of $45^{\circ}$ acquire anti-symmetric mode for LCPL irradiation and symmetric mode for RCPL irradiation, which causes the largest energy difference and therefore shows the strongest chiroptical response among a series of different interlayer angles.

We sought, therefore, to experimentally build a two-layered Ag NW assembly structure. We first synthesized Ag NWs with an average diameter of $60 \mathrm{~nm}$ (as simulated) and length of $15 \mu \mathrm{m}$, via a strategy of hydrothermal-based nucleation and growth of nanocrystals (Figs S6 and S7). We then used a developed LB technique to fabricate desired 2D monolayer ordered Ag NWs (Fig. S8) via isotropic compressing, followed by transferring the NW assembly to the quartz substrate as the first-layer of the final chiral film. Before covering the second layer of aligned NWs on the substrate, we rotated the quartz plate anticlockwise with angles of $15^{\circ}, 30^{\circ}, 45^{\circ}, 60^{\circ}, 75^{\circ}$, and $90^{\circ}$, respectively, and thus obtained six types of left-handed twolayered Ag NW films.

We characterized the resulting Ag-NW-based films, including disordered one-layer, ordered one-layer, and aligned two-layers with different interlayer angles using SEM, and found almost all NWs are well arranged in the designed ways after assembly operations (Fig. 3x-1 and Fig. S9). The polarization transmission spectra (Fig. 3c and Fig. S10) and the polar coordinates of the
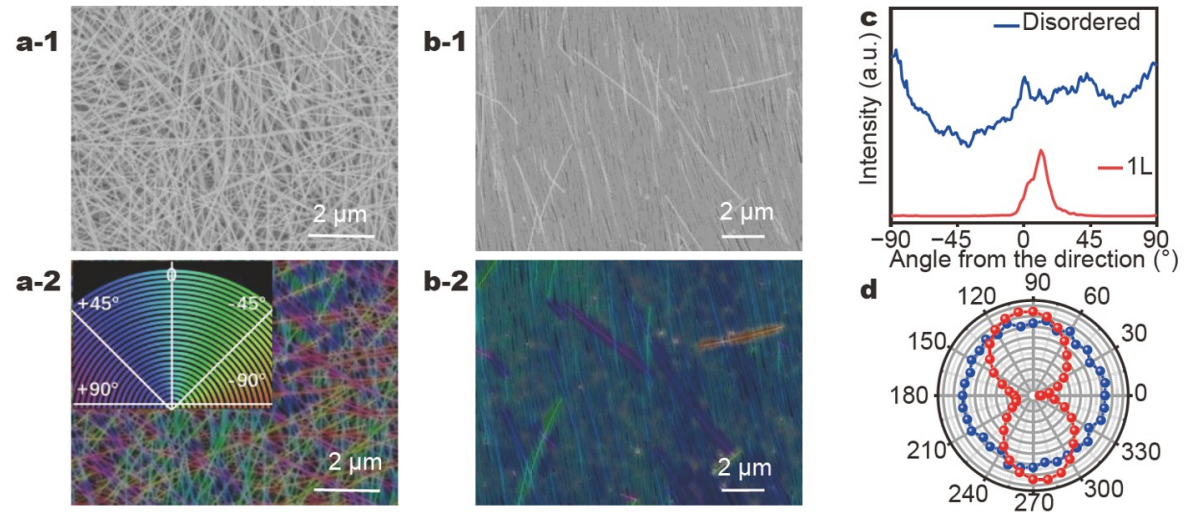

e-1

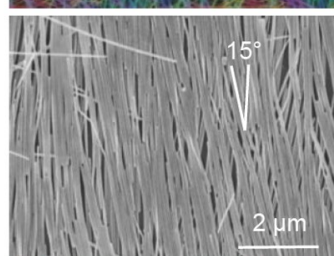

f-1
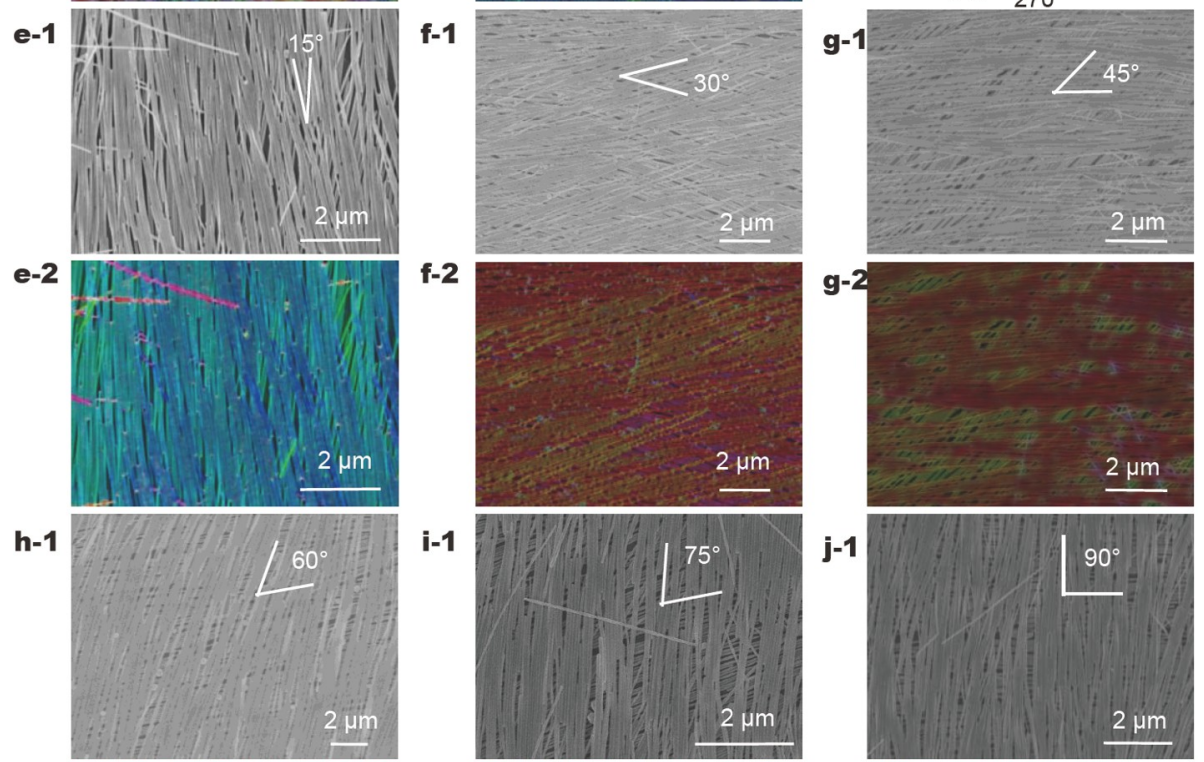

f-2
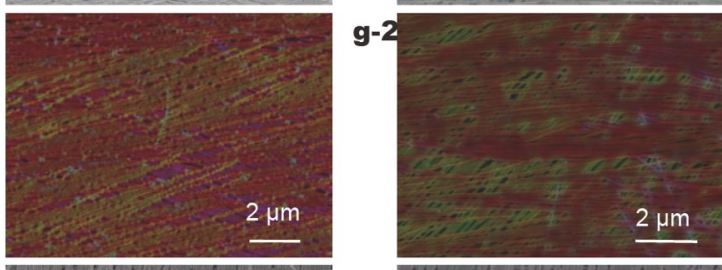

i-1
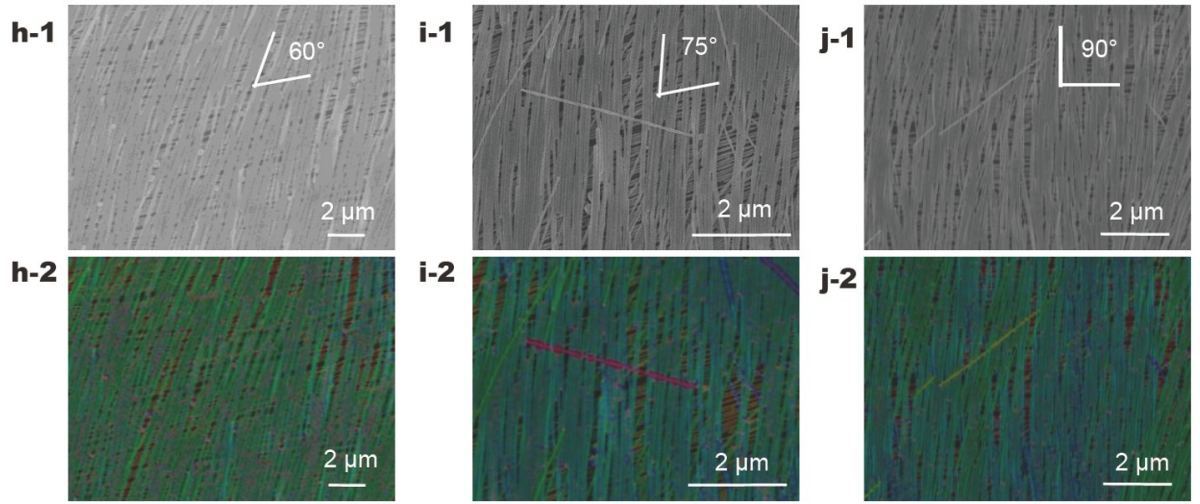

Figure 3 Characterization of one-layered and two-layered Ag NW films. (a, b) SEM images ( $x-1)$ and color-coded SEM images ( $x-2)$ related to the orientation distributions of disordered (a) and ordered one-layered (b) Ag NW films. The inset of (a-2) is circular color map coding. (c) Corresponding angledependent intensity distributions of disordered (blue curve) and ordered (red curve) one-layered films, obtained from Image- $J$ software. (d) Polar diagram of polarization-dependent transmission intensity of disordered (blue curve) and ordered (red curve) one-layered films. (e-j) Characterization of two-layered films with interlayer angles of $15^{\circ}(\mathrm{e}), 30^{\circ}(\mathrm{f}), 45^{\circ}(\mathrm{g}), 60^{\circ}(\mathrm{h}), 75^{\circ}(\mathrm{i})$, and $90^{\circ}(\mathrm{j})$, respectively. 
polarization-dependent transmission intensity (Fig. 3d) further evidenced the orderliness. The color distribution maps (Fig. 3x-2), corresponding to each SEM image (Fig. 3x-1) obtained by Image- $J$ software, showed the NW assembling orientations and the relative angles between two adjacent layers, which was verified by different colors.

We then examined the chiroptical property of Ag assembly structures with different interlayer angles from $15^{\circ}$ to $90^{\circ}$ using CD absorption spectrometer. For each sample, we tested several times with the substrate rotated by a certain angle perpendicular to the optical path direction to exclude linear dichroism signal from CD (Fig. S11). At proper interlayer angles $\left(15^{\circ}-75^{\circ}\right)$, the intensity of chiral optical activity exhibits a positive parabolalike path, with a maximum at $45^{\circ}$ (Fig. $4 \mathrm{a}$, b), well-agreeing with the prediction; for an interlayer angle of $90^{\circ}$, the structure is achiral and the result shows nearly zero chiral activity as expected. We achieved a CD of up to 1800 mdeg at $390 \mathrm{~nm}$ with the corresponding $g$-factor average value of 0.03 (Fig. S12). Through testing other two-layered films constructed by tuning Ag NW diameters, we further confirmed that Ag NWs with the diameter of $60 \mathrm{~nm}$ display the strongest signal (Fig. S13).

We further manipulated the chirality direction towards the multifunctional or specific applications via tuning the rotation of the second-layer-aligned assembly relative to the first-layer (Fig. 4c). On contrary to the left-handed film fabrication, we transferred the second layer of the NW assembly after rotating the quartz plate covered with the first layer in the clockwise direction and received the right-handed structure. We tested the two-layered right-handed sample with the interlayer angle of $45^{\circ}$, showing almost mirror-symmetrical distribution with the left-handed membrane in the $\mathrm{CD}$ spectrum and $g$ value curve (Fig. 4d, e).

To optimize the film thickness for strong chiroptical activity, we designed and prepared a series of left-handed (Fig. 4f) and right-handed (Fig. S14) chiral assembly films with different layers and the controlled angle of $45^{\circ}$ between two adjacent layers. We tested the $\mathrm{CD}$ response (Fig. 4g) and UV-vis absorption (Fig. S15), and calculated the $g$ factor (Fig. 4 h) of both handedness chiral structures with different layers. The experimental results showed that the $g$ factor of the two-layered $\mathrm{NW}$ assembly structure was the highest. We further placed the sample under ambient conditions to evaluate the stability of our prepared Ag NW chiral film and found that the $g$-factor can retain over 100 days (Fig. 4i).

\section{CONCLUSIONS}

In summary, we present a bottom-up method using the LB technique to fabricate chiral inorganic nanomaterials via constructing spirally packaging Ag NW assemblies. FDTD simulations, NW structure characterizations, and CD optical
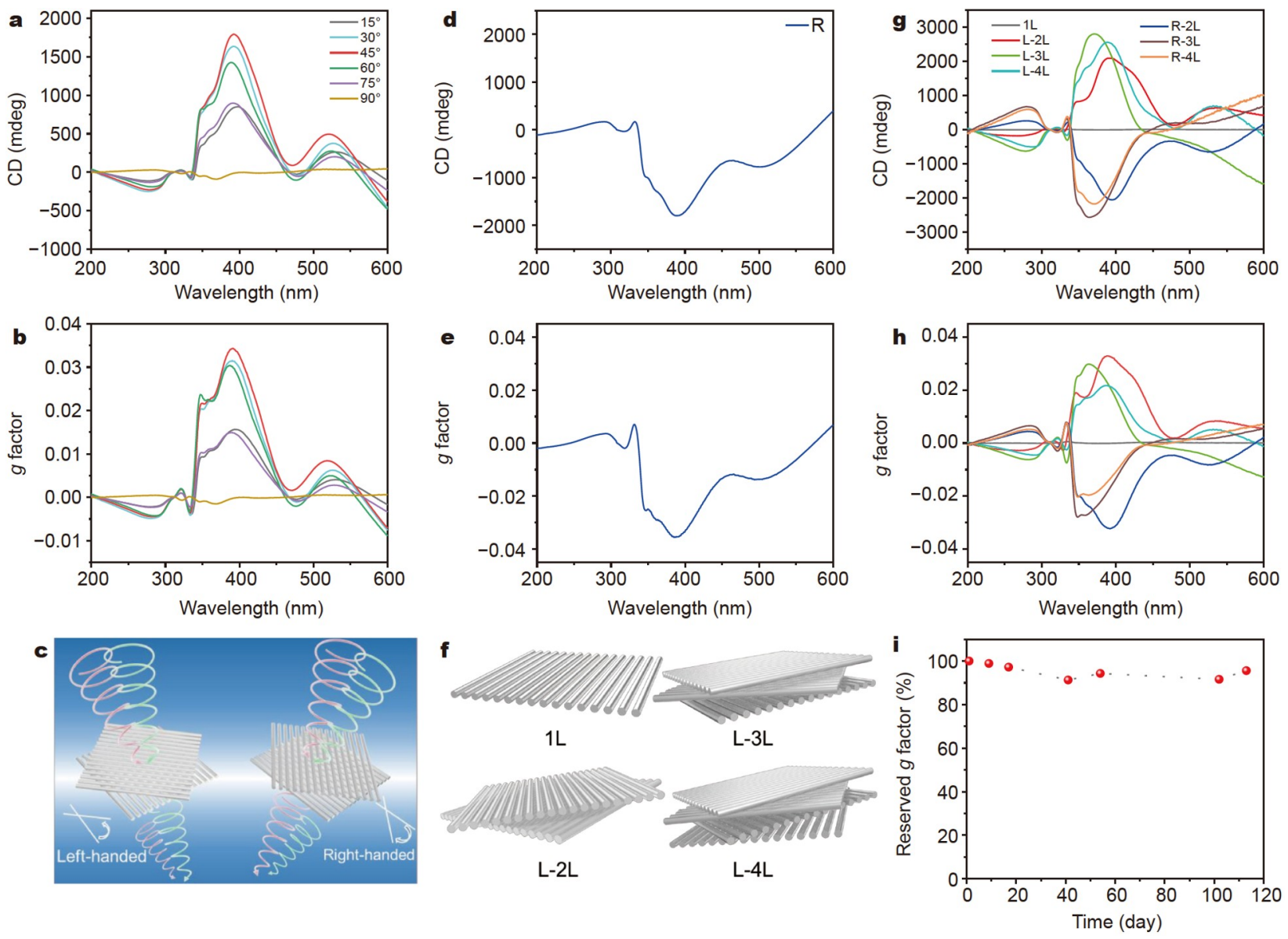

Figure 4 Chiroptical activity measurements. ( $a, b)$ CD response (a) and $g$-factor (b) of left-handed films with different interlayer angles. (c) Schematic of chirality direction manipulation. (d, e) CD spectrum (d) and $g$-factor (e) of right-handed two-layered film with the interlayer angle of $45^{\circ}$. (f) Schematics for left-handed films with different layers when fixing the interlayer angle at $45^{\circ}$. (g, h) CD spectra $(\mathrm{g})$ and $g$-factor (h) of left-handed and right-handed films with different layers. (i) The $g$-factor change when placing the film in an ordinary box for 113 days. 
measurements prove the roles of the helical stacking shape, the interlayer angle between two adjacent layers and the thickness of films in tuning the chiroptical activity. These findings may offer a promising avenue for the design of chiral sensing devices using solo NW assemblies in the future.

\section{Received 5 October 2021; accepted 15 November 2021; published online 7 January 2022}

1 Zhuang TT, Li Y, Gao X, et al. Regioselective magnetization in semiconducting nanorods. Nat Nanotechnol, 2020, 15: 192-197

2 Yeom J, Yeom B, Chan $\mathrm{H}$, et al. Chiral templating of self-assembling nanostructures by circularly polarized light. Nat Mater, 2015, 14: 66-72

3 Yeom J, Santos US, Chekini M, et al. Chiromagnetic nanoparticles and gels. Science, 2018, 359: 309-314

4 Zheng H, Li W, Li W, et al. Uncovering the circular polarization potential of chiral photonic cellulose films for photonic applications. Adv Mater, 2018, 30: 1705948

$5 \mathrm{Lu} \mathrm{J}$, Xue Y, Bernardino K, et al. Enhanced optical asymmetry in supramolecular chiroplasmonic assemblies with long-range order. Science, 2021, 371: 1368-1374

6 Liu J, Mukherjee S, Wang F, et al. Homochiral metal-organic frameworks for enantioseparation. Chem Soc Rev, 2021, 50: 5706-5745

7 Fraile JM, García JI, Herrerías CI, et al. Enantioselective catalysis with chiral complexes immobilized on nanostructured supports. Chem Soc Rev, 2009, 38: 695-706

8 Wang G, Hao C, Ma W, et al. Chiral plasmonic triangular nanorings with SERS activity for ultrasensitive detection of amyloid proteins in Alzheimer's disease. Adv Mater, 2021, 33: 2102337

9 Probst PT, Mayer M, Gupta V, et al. Mechano-tunable chiral metasurfaces via colloidal assembly. Nat Mater, 2021, 20: 1024-1028

$10 \mathrm{Kim}$ YH, Zhai Y, Lu H, et al. Chiral-induced spin selectivity enables a room-temperature spin light-emitting diode. Science, 2021, 371: 11291133

11 Lee YY, Kim RM, Im SW, et al. Plasmonic metamaterials for chiral sensing applications. Nanoscale, 2020, 12: 58-66

$12 \mathrm{Wu}$ T, Kessler J, Bour P. Chiral sensing of amino acids and proteins chelating with $\mathrm{Eu}^{\mathrm{III}}$ complexes by Raman optical activity spectroscopy. Phys Chem Chem Phys, 2016, 18: 23803-23811

13 Hug W. Visualizing Raman and Raman optical activity generation in polyatomic molecules. Chem Phys, 2001, 264: 53-69

14 Abdali S, Blanch EW. Surface enhanced Raman optical activity (SEROA). Chem Soc Rev, 2008, 37: 980-992

15 Liu Z, Ai J, Kumar P, et al. Enantiomeric discrimination by surfaceenhanced Raman scattering-chiral anisotropy of chiral nanostructured gold films. Angew Chem Int Ed, 2020, 59: 15226-15231

16 Funston AM, Novo C, Davis TJ, et al. Plasmon coupling of gold nanorods at short distances and in different geometries. Nano Lett, 2009, 9: 1651-1658

17 Rycenga M, Cobley CM, Zeng J, et al. Controlling the synthesis and assembly of silver nanostructures for plasmonic applications. Chem Rev, 2011, 111: 3669-3712

18 Hu H, Sekar S, Wu W, et al. Nanoscale bouligand multilayers: Giant circular dichroism of helical assemblies of plasmonic 1D nano-objects. ACS Nano, 2021, 15: 13653-13661

19 Auguié B, Alonso-Gómez JL, Guerrero-Martínez A, et al. Fingers crossed: Optical activity of a chiral dimer of plasmonic nanorods. J Phys Chem Lett, 2011, 2: 846-851

20 Yang C, Gu H, Lin W, et al. Silver nanowires: From scalable synthesis to recyclable foldable electronics. Adv Mater, 2011, 23: 3052-3056

21 He Z, Chang LG, Lin Y, et al. Real-time visualization of solid-phase ion migration kinetics on nanowire monolayer. J Am Chem Soc, 2020, 142: 7968-7975

22 Li B, Ye S, Stewart IE, et al. Synthesis and purification of silver nanowires to make conducting films with a transmittance of $99 \%$. Nano Lett, 2015, 15: 6722-6726

23 Lv J, Hou K, Ding D, et al. Gold nanowire chiral ultrathin films with ultrastrong and broadband optical activity. Angew Chem Int Ed, 2017,

\section{6: $5055-5060$}

24 Lv J, Ding D, Yang X, et al. Biomimetic chiral photonic crystals. Angew Chem Int Ed, 2019, 58: 7783-7787

25 Yin X, Schäferling M, Metzger B, et al. Interpreting chiral nanophotonic spectra: The plasmonic Born-Kuhn model. Nano Lett, 2013, 13: 6238-6243

26 Han C, Yang L, Ye P, et al. Three dimensional chiral plasmon rulers based on silver nanorod trimers. Opt Express, 2018, 26: 10315-10325

27 Zhao SX, Zhang W. Plasmonic chirality of one-dimensional arrays of twisted nanorod dimers: The cooperation of local structure and collective effect. Opt Express, 2019, 27: 38614-38623

28 Manjavacas A, García de Abajo FJ. Coupling of gap plasmons in multiwire waveguides. Opt Express, 2009, 17: 19401-19413

Acknowledgements This work was supported by the National Natural Science Foundation of China (22071226, 51732011, 21431006, 21761132008, 81788101,11227901 and 11874164), the Funding of University of Science and Technology of China (KY2060000168 and YD2060002013), the Foundation for Innovative Research Groups of the National Natural Science Foundation of China (21521001), the Key Research Program of Frontier Sciences, CAS (QYZDJ-SSW-SLH036), and the Users with Excellence and Scientific Research Grant of Hefei Science Center of CAS (2015HSC-UE007). The authors thank Prof. Mingming Ma's group at the University of Science and Technology of China for circular dichroism absorption spectrum test.

Author contributions Zhuang T and Yu SH supervised the project. Pang Y supervised the simulations. Zhuang $\mathrm{T}$ and Guo $\mathrm{X}$ conceived the idea and wrote the paper. Guo X carried out the experiments. Guo X and Li Y collected and analyzed the data. Wu D carried out the simulations. He Z, Wang JL, Zhang $\mathrm{C}$ and Pan $\mathrm{Z}$ helped to characterize the material structures. All authors discussed the results and assisted in manuscript preparation.

Conflict of interest The authors declare that they have no conflict of interest.

Supplementary information Supporting data are available in the online version of the paper.

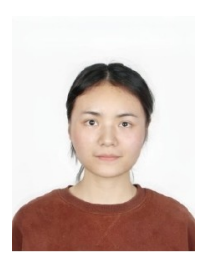

Xueru Guo received her BS degree from the College of Chemistry and Materials Science, Anhui Normal University in 2020. She is studying for a master's degree at the Department of Chemistry, University of Science and Technology of China (USTC) under Prof. Taotao Zhuang's supervision. Her research focuses on the chiral structure and function.

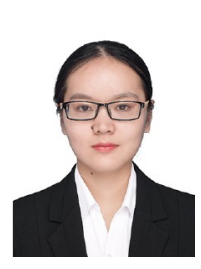

Dan Wu received her BS degree from the School of Optical and Electronic Information, Huazhong University of Science and Technology. She is currently a PhD candidate in the group of Prof. Pang at Huazhong University of Science and Technology. Her research focuses on the assembling of single-photon sources using nano-optical tweezers, and the integration of such sources into all-fiber systems.

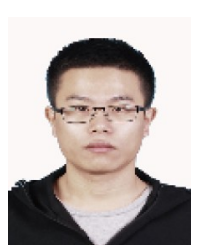

Yi Li received his $\mathrm{PhD}$ degree under the supervision of Prof. Shu-Hong Yu at USTC in 2019. From 2019 to 2021, he worked as a postdoctoral fellow in Prof. Shu-Hong Yu's group. He is currently a research associate at USTC. His research focuses on the synthetic chemistry, photophysics, and applications of novel one-dimensional semiconductor nanostructures. 


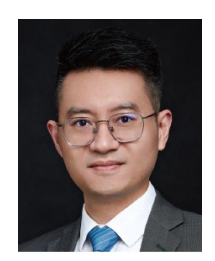

Yuanjie Pang received his $\mathrm{PhD}$ degree from the Department of Electrical and Computer Engineering of the University of Victoria in 2012. He was a postdoctoral fellow at the University of Michigan during 2012-2015, and a postdoctoral fellow appointed by the University of Toronto during 2015-2018. He was appointed as a professor at Huazhong University of Science and Technology in 2018. His research interests include nano-optical tweezers and renewable energy storage.

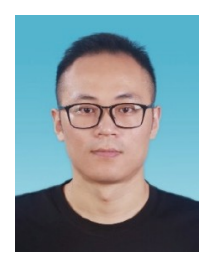

Taotao Zhuang received his $\mathrm{PhD}$ degree in inorganic chemistry from USTC and then worked as a postdoc fellow at the University of Toronto. He was appointed as a professor in 2020 at USTC. Now he is trying to synthesize new inorganic chiral nanomaterials, understand the mechanism of structure-property, and realize the practical applications using homemade materials.

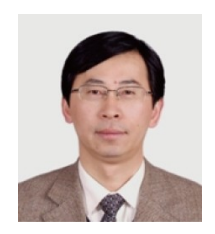

Shu-Hong Yu completed his PhD degree from USTC. In 2002, he was appointed as the Cheung Kong Professor. Currently, he leads the Division of Nanomaterials \& Chemistry at Hefei National Laboratory for Physical Sciences at the Microscale, USTC. He was elected as an academician of Chinese Academy of Sciences in 2019. His research interests include bio-inspired synthesis and selfassembly of nanostructured materials and nanocomposites, and their related properties.

\section{银纳米线有序化实现手性光学活性}

郭雪儒 ${ }^{\dagger \dagger}$, 吴丹 ${ }^{2 \dagger}$, 李毅 ${ }^{1 \dagger}$, 何振 ${ }^{1}$, 王金龙 ${ }^{1}$, 张崇 ${ }^{1}$, 潘㻇 $^{1}$, 庞元杰 ${ }^{2 *}$, 庄涛涛 ${ }^{*}$, 俞书宏 ${ }^{*}$

摘要 随着数十年的研究, 手性材料在生物、化学、物理等领域的应 用取得了显著进展. 本文报道了一种基于纯银纳米线、自下而上的手 性构建策略, 用以制备在紫-蓝光能带中具有较强光学活性的手性等离 子体薄膜. 时域有限差分模拟预测了一定层间角的银纳米线螺旋结构 具有较强的手性光学活性. 我们采用Langmuir-Blodgett技术通过调节 层间角度和方向, 构建了具有左、右旋手性的层状纳米线组装结构. 银 纳米线组装体的圆二色性强度与层间角和膜厚相关, 其不对称因子最 高可达 0.03 . 这些新的手性结构由于特殊的等离子特性, 将成为手性传 感器的理想主体材料. 